\title{
Noninvasive infrared analyses for brain-to-breast desease by monitoring angle-coherent spectra from photonic quantum ring laser of whispering gallery modes [Invited]
}

\author{
O'D. Kwon ${ }^{1, *}$, K. S. Yoon ${ }^{1}$, J. S. Lee ${ }^{1}$, and Y. Chung ${ }^{1}$ \\ ${ }^{1}$ Elec. Eng. Dept., Pohang University of Science \& Technology [POSTECH], Pohang, KB 37673 Korea [ROK] \\ *odkwon@postech.ac.kr
}

\begin{abstract}
Noninvasive and portable diagnoses via angle-resolved coherent spectral data are presented. ACTIRS (Angleresolved coherent tomographic IR spectroscopy) through angle-coherent return spectra from photonic quantum ring laser of whispering gallery modes, which is not photocurrent-converted after the cortical, vascular or muscular diffuse paths, can be a new method for brain/heart studies. Also it will be eligible for frequent do-ityourself diagnoses of breast cancer before hospital visits. The unique angle-coherent multi-spectra will eventually lead to study ionic or protein contents of cranial, arterial or lactiferous ducts that have been obtained only from uncomfortable biopsy, NMR, CT or mammography. The spectral data can be spatially or temporally improved and correlated for extracting more meaningful diagnostic conclusions
\end{abstract}

Keywords: Photonic quantum ring (PQR); MQW; ACTIRS; WGM; whispering cave mode; secondary brain injury (SBI); breast cancer

\section{PQR laser chip fabrication and new spectral methodolo gy :ACTIRS}

Photonic quantum ring (PQR) laser, a cylindrical mesa structure of active Ga(Al)As multi-quantum well (MQW) plane between top and bottom distributed Bragg reflectors (DBRs), functions as concentric quantum-wire emitters within the PQR's peripheral Rayleigh band of 2-dimensional (2D) MQW circles, which is associated by nature with traditional 2D whispering gallery mode (WGM) phenomena in $\mathrm{x}-\mathrm{y}$ plane as explained by Lord Rayleigh in 1912. The PQR Rayleigh band plane is now surrounded by new 3D whispering cave mode (WCM) region, which means resonant helical travelling-waves within the imaginary PQR toroid [1] (Figure 1). The systematic variations of intermodal spacing agree with a well-defined angular quantization rule (AQR) [2,3] (Figure 2).
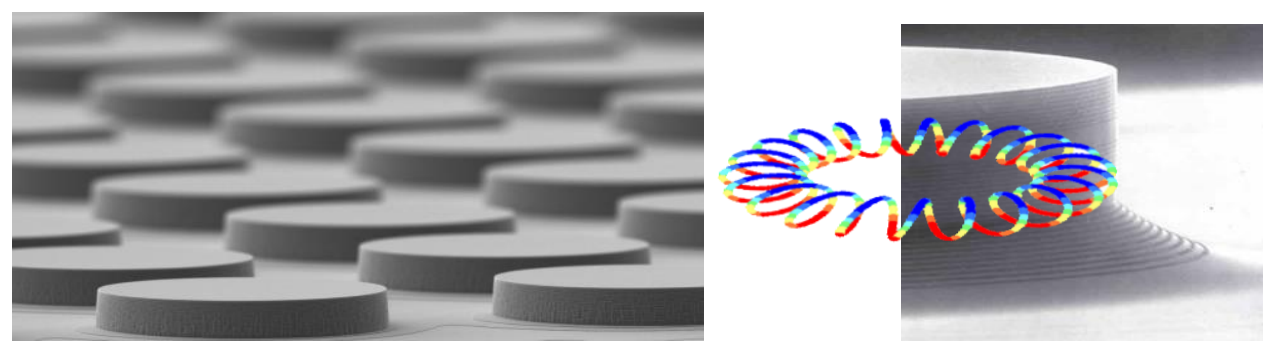

Figure 1. SEM picture of 1K PQR mesas; 3D Helical travelling waves of the PQR toroid around 2D active plane 
of central MQWs.
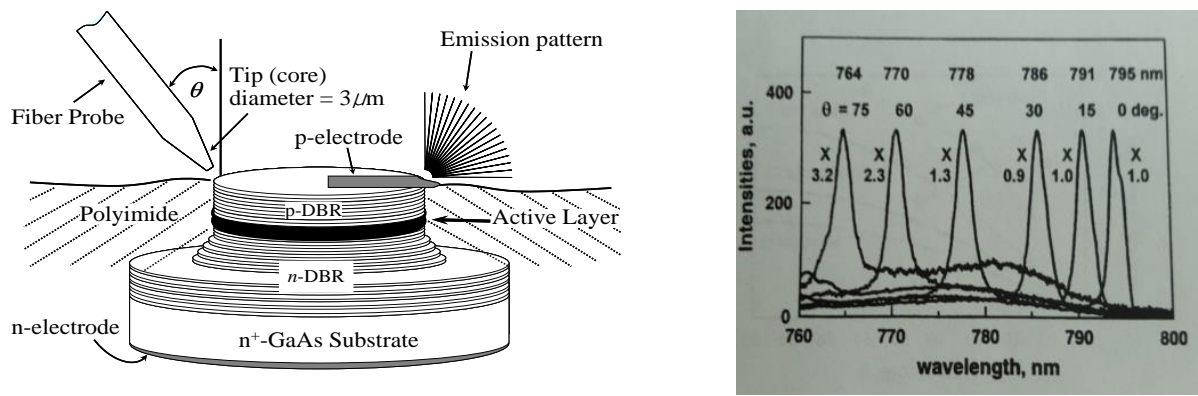

Figure 2. Inclination or zenith angle-dependent measurement setup; PQR's angle-resolved tunable frequency ranges.

The PQR laser thresholds (active diameters around 20-30 $\mu \mathrm{m}$ ) are in a few tens of micro-amperes as predicted by a quantum wire-like threshold formula [1] while remaining stable up to above $130^{\circ} \mathrm{C}$, and normally operated well below thresholds of vertical cavity surface emitting lasers (VCSELs) similar in size. The PQR lasers usually exhibit 3D spectra of zenith-angle-governed blue-shifts in the $20 \mathrm{~nm}$ range, implying that it is possible to extend systematically the PQR's tunable frequency ranges in $20 \mathrm{~nm}$ steps from around 700 to $980 \mathrm{~nm}$ using a group of different $\mathrm{PQR}$ device chips fabricated with various compound semiconductors from (Al)GaAs or (In)GaAs, to cover all required ionic IR frequency ranges within the transparent spectral window between water and hemoglobin barriers (Figure 2/right).

In fact the AQR gives rise to various inclination angle of helical waves of the PQR toroid, directly defined through air-tissue-polyimide-PQR semiconductor interfacial refractions. They correspond to the angle-coherent spectral properties, which then become a passageway to develop the angle-resolved coherent tomographic infrared spectroscopy (ACTIRS), whose outgoing light wavelengths to the target tissue are now distributed around the $\mathrm{z}$-axis with various zenith angles via AQR. The return light signals can be detected with an optical fiber probe (multi-fiber probes at selected angles may be preferable for multi-target points), adjusted to selected angles and coupled to an inexpensive portable spectrum analyzer(PSA), e.g., HR4000/Ocean Optics model for personal preventive use [or for higher performance, e.g., HP70004A optical spectrum analyzer(OSA) for expensive professional analysis]. We stress here that light is never photocurrent-converted after diffuse paths in order to preserve return spectra information. This is the last key difference from conventional near infrared spectroscopy (NIRS) limited to the photocurrent-used oxygenation studies.

\section{Experimental examples toward brain and breast diagnoses}

\subsection{Reflection Study}

Active mesas of 80- $\AA \mathrm{Al}_{0.1} \mathrm{Ga}_{0.9}$ As multi-quantum wells between 8-pair top and 40-pair bottom $\mathrm{Al}_{0.9} \mathrm{Ga}_{0.1}$ As / $\mathrm{Al}_{0.3} \mathrm{Ga}_{0.7}$ As DBR structures, $25 \mu \mathrm{m}$ in diameter, or the PQR lasers were integrated to fabricate a $1 \mathrm{~K} P Q R$ chip (16x16x4). Each laser will create angle-resolved coherent $(\mathrm{AC})$ resonances within the PQR toroid, which produces resonant double helix multiple waves ( $\mathrm{CW}$ and $\mathrm{CCW}$ ) inside the toroid via $\mathrm{AQR}$, generating blueshifted multimode tails together with the main pulse with approximately $780 \mathrm{~nm}$ spectral peak (red in Figure 3/left). The $1 \mathrm{~K} P Q R$ chip laser was normally incident to the human cortical (frontal lobe) region, while the return spectra, blue and green, collected (two monitoring points, normal and off-normal 30 degree) with a fiber probe for the backward response through cortical diffuse paths. We used HR4000 PSA here, which means blurred spectra. Improved spectral resolutions are expected with expensive and delicate HP70004A OSA. Very fine sub-mode spectra and blue-shifted envelopes were observed with the OSA although the work was on single 
PQR (Figure 4/left). Also one may fabricate $4 \mathrm{~K}, 16 \mathrm{~K}$ or $64 \mathrm{~K}$ PQR chips for better results while the actual detection area and distance to the tissue target will be adjusted as the chip size and focusing region grows. We also observed a small but actual blue-shifted return spectrum from experiments with $1 \mathrm{~K}$ GaAs PQR chip of $850 \mathrm{~nm}$ spectral peak, vertically incident to the cortical (frontal lobe) region, while monitoring the spectra with a portable fiber probe in different directions, normal and off-normal as explained above, for the backward response through the cortical diffuse paths (Figure 4/right). The spectra observed for the first time in such a simple manner are quite surprising with symmetrically distinctive and delicate features. At the moment we are not able to tell which diffuse paths the angle-resolved beams were taking, and further work is needed with larger chips and spatiotemporal correlations.
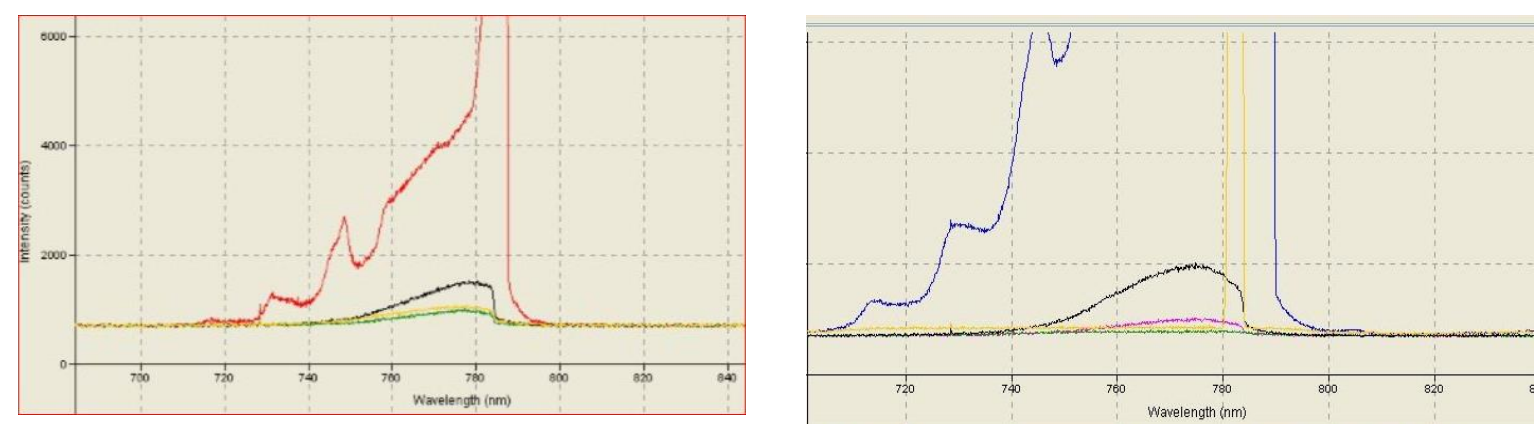

Figure 3. Left-1K AlGaAs PQR chip laser is normally incident to the frontal lobe (red). Return spectra [Reflection from frontal lobe], blue and green, (normal and off-normal 30 degree); Right-1K PQR chip laser behind an earlobe [Transmission], and the results (Figure 3/right) are approximately similar to the left curve. (Yellow peak without tail is an example observed from another pulse,) Now the output spectra transmitted through earlobe (black and red) are similar and broad while the black spectral center is blue-shifted a bit conspicuously.
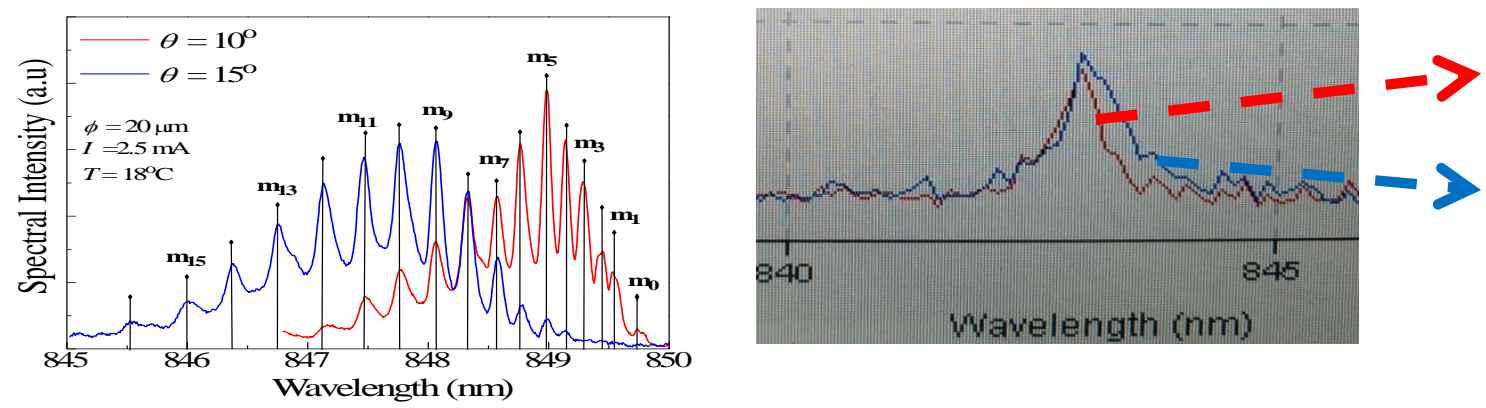

Figure 4. Left- Angular quantization rule (AQR) representing a series of discrete sub-modes whose spectral envelope peaks are gradually blue-shifted; Right- backward response through the cortical diffuse paths. The spectra with symmetrically distinctive features: Red curve ( 30 degree off-normal) shows a blue-shift with respect to the blue curve (normal).

In advance we may consider an imaginary but very crucial situation where the non-invasive ACTIRS can be 
quite useful. This requires detailed explanations with some quotes of $<$ I,II,III $>$ [4]: $<$ I $>$ Multimodality monitoring (MMM) is a recently developed method that aids in understanding real-time brain physiology. Early detection of physiological disturbances is possible with the help of MMM, which allows identification of underlying causes of deterioration and minimization of secondary brain injury (SBI). MMM is especially helpful in comatose patients with severe brain injury because neurological examinations are not sensitive enough To detect SBI, the variables frequently examined in MMM are hemodynamic parameters such as intracranial pressure (ICP), cerebral perfusion pressure (CPP), and mean arterial pressure (MAP); brain specific oxygen tension; markers for brain metabolism including glucose, lactate, and pyruvate levels in brain tissue; and cerebral blood flow $(\mathrm{CBF}) ;<\mathrm{II}>$ Now, hemorrhagic complication risk stemming from MMM probe placement is reported to be similar to that stemming from extra-ventricular drain (EVD) insertion. Most reported cases with probe-related infection had concomitant EVD, and infection is ascribed to be associated with EVD insertion, rather than direct infection via burr-hole procedure. Currently, the MMM probe is inserted visually while targeting the perilesional tissue. However, more studies are needed to clarify the best location for monitoring ICP; $<$ III $>$ One big limitation of MMM for patients with severe stroke is its invasiveness. Currently, a noninvasive but accurate monitoring technology does not exist. Moreover, the location of the probe itself is still not standardized. Probes are inserted into the brain tissues at high risk of SBI and therefore, mostly targeted at the perilesional or penumbra area.

Because of the critical SBI situations explained above, we instead pursue the non-invasive passage of ACTIRS that can choose the cortical (C), vascular (V), or muscular (M) diffuse paths which assure us to protect return spectra information. This angle-coherent spectral method for human organ studies may be extended eventually to the ionic or protein composition analysis as well, through spatially and temporally improved and correlated techniques toward full ACTIRS for spectral identification. The improvement here could mean, for instance, multi-target $(\mathrm{C} 1 \& \mathrm{~V} 1)$ points, or multi-target $(\mathrm{V} 1 \& \mathrm{~V} 2)$ correlation time-delay points. The time-delay responses together with pulsation cycles will serve as some various velocity indicators leading to local pressure variations via Bernoulli's principle for detecting local ICP or MAP variations that could tell and monitor the SBI status.

\subsection{Transmission Study}

The $1 \mathrm{~K}$ PQR chip laser was then placed behind an earlobe for transmission studies, and the results (Figure 3/right) are approximately similar to Figure 3/left: the input spectral shape (blue in Figure 3/right) also generates blue-shifted tails. (Yellow peak without tail is an example observed from another pulse,) Now the output spectra transmitted through earlobe (black and red) are similar and broad while the black spectral center is blue-shifted a bit conspicuously. For detailed ACTIRS, PQR chips and detection S/W improvements will help a great deal for the powerful future of noninvasive and portable diagnoses.

In particular, the present transmission studies can be very convenient and simple techniques for personal and comfortable do-it-yourself diagnoses of breast cancer as early check-up before hospital visit for intensive mammographic inspections and biopsy. The above $1 \mathrm{~K}$ chip performance however suggests that $4 \mathrm{~K}-16 \mathrm{~K} \mathrm{PQR}$ chips assisted with improved detection methods will do the job much better since the dangerous breast portion is frequently lumps in lactiferous ducts, and usually accessible for mammographic setup while the region is larger than earlobe. The personal medical instrument however will be battery-operated and can remotely transmit brief datasheets to the hospital.

\section{Conclusions}

Angle-coherent spectral treatment will be useful for human organism studies: today diagnosis tomorrow treatment! All researchers interested in ACTIRS can take leading roles, because every part of human body will 
be playground open to aspiring people anywhere. I trust my university which has supported me will be happy to see this hand-in-hand miracle as well.

Funding: This research received indirect partial POSTECH funding from Kumho.

\section{Acknowledgments}

The authors thank Y. C. Kim, M. Kwon, B. H. Park, and J. Bae for various assistances, and Profs. Chae Yong Kim and Si Un Lee, Seoul National University College of Medicine/Bundang Hospital for medical guiding.

Conflicts of Interest: The authors declare no conflict of interest.

\section{References}

1. Ahn, J.C. et al. Photonic Quantum Ring, Phys. Rev. Lett. 1999, 82, 536.; Kwon, O. et al. Photonic Quantum Ring from Quantum Corral of Whispering Cave Mode, in Trends in Nano-and Micro-Cavities, Kwon, O.; An, K.; Lee, B. Eds. 2011, 152-187, London: Bentham Sci. Publ. Ltd. e-Book.

2. Kwon, O. IEEE Photonics Conf. 2018, USA (invited); Bae, J.; Lee, J.; Kwon, O.; Minogin, V. G. Spectrum of 3D photonic quantum ring micro-disk cavities, Opt. Lett. 2003, 28, 1861.

3. Park, B. H. Chiral wave propagation manifold of the photonic quantum ring laser, Appl. Phys. Lett. 2002, 81, 580; Ahn, J.C.; Kang, H. Y.; and Kwon, O. Angle-dependent multiple-wavelength radial emissions in a toroidal microcavity: A Photonic Quantum Ring Laser, SPIE 1998, 3283, 241.

4. Ko, S. B. Multimodality Monitoring in the Neurointensive Care Unit: A Special Perspective for Patients with Stroke, J. of Stroke 2013, 15(2), 99. 